\title{
Uma análise dos testes in vitro de força de adesão em Ortodontia*
}

\author{
Julio Orrico de Aragão Pedra e CAL NETO**, José Augusto Mendes MIGUEL***
}

\begin{abstract}
Resumo
O objetivo deste estudo foi analisar as metodologias empregadas em testes in vitro de força de adesão em Ortodontia. Foram selecionados nos periódicos American Journal of Orthodontics and Dentofacial Orthopedics e The Angle Orthodontist (do ano de 1993 até 2002) todos os artigos onde foi avaliada a resistência de adesão de braquetes a diferentes superfícies, através de Máquinas de Ensaio Universal Instron ou similares. Foram analisados 127 artigos, e destes $86 \%$ foram conduzidos através de ensaios de cisalhamento, ao passo que $14 \%$ foram através de ensaios de tração. Dentre os estudos com dentes humanos (68\%), os pré-molares foram os mais utilizados (57\%), seguidos pelos molares (31\%). Quanto ao número de espécimes por grupo, 28 estudos analisaram entre 6 e 10; 30 entre 11 e 15; 33 entre 16 e 20; e 36 analisaram mais de 20 espécimes. Como solução de armazenamento, as mais empregadas foram: água destilada (43\%), seguido por Timol a 0,1\% (28\%) e Cloreto de sódio a $0,9 \%(12 \%)$. No que diz respeito à velocidade de operação da máquina, em 25 estudos foi de $0,5 \mathrm{~mm} / \mathrm{min}$; em 43 foi de $1,0 \mathrm{~mm} / \mathrm{min}$; em 8 foi de $2,0 \mathrm{~mm} / \mathrm{min}$; e em 38 foi de $5,0 \mathrm{~mm} / \mathrm{min}$. Os autores concluem que não existe um consenso na metodologia de tais estudos, sendo então evidenciada a necessidade de uma possível padronização da técnica.
\end{abstract}

Palavras-chave: Colagem Ortodôntica. Compósitos Resinosos. Ortodontia.

\section{INTRODUÇÃO}

A colagem direta de braquetes é considerada uma das evoluções mais significativas nos últimos anos da terapia ortodôntica fixa ${ }^{12,14,15}$. A partir da técnica do condicionamento ácido apresentada por Buonocore ${ }^{3}$, foi desenvolvida uma gama de materiais adesivos, sendo a resina composta a mais empregada para a colagem em Ortodontia.

Quando melhorias em produtos surgem, os níveis de força de adesão freqüentemente estão entre as principais vantagens anunciadas, de forma que a determinação da força de adesão in vitro continua sendo de grande importância e interesse.

$\mathrm{Na}$ procura por materiais cada vez mais adequados, os estudos laboratoriais e clínicos são vitais para a indicação definitiva dos novos produtos. A literatura tem apresentado inúmeros relatos de testes de adesão realizados em Ortodontia. Um problema é que as avaliações nem sempre são facilmente interpretadas.

Ao observar a força de adesão de braquetes metálicos ao esmalte, em ambiente seco e em en- 
saio de cisalhamento, proporcionada pelo uso do ácido fosfórico associado ao Transbond XT (3M Unitek, Monróvia, Califórnia), Webster et al. ${ }^{18}$ encontraram valores discrepantes de Bishara et al. ${ }^{2}$ (26,9 Mpa vs 10,4 Mpa). Quais seriam as possíveis razões para isso? Talvez as diferenças na condução dos experimentos. No primeiro trabalho foram utilizados dentes bovinos, com 12 espécimes por grupo, condicionados por 60 segundos, sendo descolados mediante uma velocidade da célula de carga de $0,5 \mathrm{~mm} / \mathrm{min}$. Já no segundo trabalho, foram utilizados molares humanos, com 20 espécimes por grupo, condicionados por 30 segundos, sendo descolados por ação da célula de carga cuja velocidade era de $5 \mathrm{~mm} / \mathrm{min}$.

Devido à grande diversidade de materiais e métodos empregados em tais estudos para teste de diferentes materiais, comparações entre resultados obtidos por diferentes pesquisadores têm se tornado cada vez mais inviáveis.

Fox, Mc Cabe e Buckley ${ }^{6}$ realizaram uma revisão de 66 artigos que avaliavam a força de adesão in vitro de acessórios ortodônticos à superfície de esmalte dentário. Foi observado que não houve um consenso quanto à metodologia empregada, sendo evidenciada a necessidade de uma padronização dos procedimentos, e conseqüentemente, da elaboração de um protocolo universal, a fim de que comparações possam ser realizadas de forma mais coerente, oferecendo maior confiabilidade ao clínico.

O objetivo deste estudo foi analisar as metodologias empregadas recentemente em testes in vitro de força de adesão em Ortodontia e verificar se persiste a necessidade de uma padronização de procedimentos.

\section{MATERIAIS E MÉTODOS}

Foi realizada uma busca sobre testes de força de adesão in vitro em Ortodontia, em dois dos principais periódicos disponíveis na área, American Journal of Orthodontics and Dentofacial Orthopedics e The Angle Orthodontist, nos últimos dez anos (1993 a 2002). Devido à grande quantidade de publicações sobre este tema, foram utilizados os seguintes critérios de inclusão:

1) Deveria haver braquetes colados de forma direta a diferentes superfícies.

2) Deveriam ser comparados ao menos dois grupos.

3) Os testes deveriam ser conduzidos por meio de Máquinas de Ensaio Universal Instron ou similares.

\section{Os seguintes dados foram coletados de cada artigo selecionado}

1) O tipo de substrato utilizado para colagem.

2) A solução adotada para armazenamento dos espécimes.

3) A direção da força aplicada para descolagem durante o ensaio.

4) O número de espécimes por cada grupo utilizado no teste.

5) O tipo de braquete utilizado no teste.

6) A unidade utilizada para quantificar a força de adesão.

7) A velocidade da célula de carga adotada para o ensaio.

8) O método para avaliação da resina remanescente.

9) A análise estatística utilizada para a comparação dos grupos.

\section{RESULTADOS}

Como resultado da busca, foram selecionados 127 artigos que se enquadraram nos critérios de inclusão e constituíram a amostra deste estudo.

\section{O tipo de substrato utilizado para colagem}

A tabela 1 apresenta a distribuição dos diferentes substratos adotados para testes de força de adesão em Ortodontia ao longo dos anos. Foi observado que os dentes humanos continuam a ser os mais utilizados (68\%), embora tenha havido uma grande utilização de dentes bovinos (19\%). A tabela 2 mostra a distribuição quanto ao tipo de dente adotado, dentre os estudos onde dentes humanos permanentes foram utilizados como subs- 
trato. Apesar de haver uma grande variação, os prémolares são os dentes mais comumente utilizados (57\%), sendo seguidos pelos molares (31\%).

\section{Soluções para armazenamento dos espécimes}

A tabela 3 mostra as soluções utilizadas para estocagem dos espécimes. A água destilada foi a mais comum, sendo utilizada em 55 dos artigos, seguida pelo Timol 0,1\% aparecendo em 36 artigos, enquanto em 4 deles os autores falharam no sentido de mencionar a solução utilizada.

\section{Direção da força aplicada durante a descolagem}

As direções adotadas para os ensaio de descolagem são apresentadas na tabela 4. Em 106 (84\%) artigos se optou por testar os espécimes através de ensaios de cisalhamento, ao passo que em 17 (13\%) se optou por cargas de tração e em 4 (3\%) foram realizados ensaios em diferentes direções.

\begin{tabular}{|c|c|}
\hline Substrato utilizado & Número de Publicações \\
\hline Dentes humanos permanentes & 88 \\
\hline Dentes Bovinos & 25 \\
\hline Superfícies Cerâmicas & 9 \\
\hline Amálgama & 5 \\
\hline Ouro & 2 \\
\hline Superfícies Plásticas & 1 \\
\hline Total & 130 \\
\hline
\end{tabular}

Tabela 3 - Soluções para estocagem dos espécimes.

\begin{tabular}{|l|c|}
\multicolumn{1}{|c|}{ Solução } & Número de Publicações \\
\hline Água Destilada & 55 \\
\hline Timol 0,1\% & 36 \\
Cloreto de Sódio 0,9\% & 15 \\
Cloramina T 0,5\% & 9 \\
\hline Outras & 8 \\
\hline ñ mencionada & 4 \\
\hline \multicolumn{1}{|c|}{ Total } & $\mathbf{1 2 7}$ \\
\hline
\end{tabular}

\section{Quantidade de espécimes por grupo}

O número de espécimes por cada grupo é apresentado na tabela 5. Como freqüentemente foram observados casos onde o número de espécimes variou entre grupos de um mesmo estudo, optou-se por adotar sempre o menor valor. Foram adotados quatro intervalos para agrupar os artigos, sendo mais freqüente a utilização de mais de 20 espécimes por grupo (28\%).

\section{Tipo de braquete utilizado}

A tabela 6 indica a utilização de diferentes tipos de braquetes por estudo realizado. Os braquetes mais comumente utilizados foram os metálicos (102), representando cerca de $80 \%$ dos artigos selecionados, ao passo que os braquetes plásticos foram os menos utilizados (2), representando cerca de $1,5 \%$.

\begin{tabular}{|c|c|}
\hline Tipo de dente & Número de Publicações \\
\hline Pré-molares & 50 \\
\hline Molares & 27 \\
\hline Incisivos superiores & 6 \\
\hline Pré-molares e molares & 2 \\
\hline Incisivos e pré-molares & 1 \\
\hline não mencionado & 2 \\
\hline Total & 88 \\
\hline
\end{tabular}

\begin{tabular}{|c|c|}
\hline Tipo de ensaio adotado & Número de Publicações \\
\hline Cisalhamento & 106 \\
\hline Tração & 17 \\
\hline Cisalhamento e tração & 3 \\
\hline Cisalhamento, tração e torção & 1 \\
\hline Total & 127 \\
\hline
\end{tabular}




\begin{tabular}{|c|c|}
\hline Espécimes por grupo & Número de Publicações \\
\hline 06--10 & 28 \\
\hline 11--15 & 30 \\
\hline $16--20$ & 33 \\
\hline $20+$ & 36 \\
\hline Total & 127 \\
\hline
\end{tabular}

\begin{tabular}{|c|c|}
\hline \multicolumn{2}{|c|}{ Tabela $\mathbf{6}$ - Tipo de braquetes utilizados por estudo. } \\
\hline Tipo de braquetes & Número de Publicações \\
\hline Metálicos & 102 \\
\hline Cerâmicos & 11 \\
\hline Metálicos e Cerâmicos & 8 \\
\hline Plásticos & 2 \\
\hline Metálicos e Plásticos & 2 \\
\hline Metálicos, Cerâmicos e Plásticos & 2 \\
\hline Total & $\mathbf{1 2 7}$ \\
\hline
\end{tabular}

\begin{tabular}{|c|c|}
\hline Tabela 7 - Unidades para quantificar a força de adesão. \\
\hline Unidades & Número de Publicações \\
\hline $\mathrm{Mpa}\left(\mathrm{N} / \mathrm{mm}^{2}, \mathrm{NM} / \mathrm{m}^{2}\right)$ & 111 \\
$\mathrm{~N}(\mathrm{KgF})$ & 6 \\
$\mathrm{Kg} / \mathrm{mm}^{2}$ & 5 \\
$\mathrm{Kg} / \mathrm{cm}^{2}$ & 4 \\
$\mathrm{Kg}$ & 1 \\
Total & $\mathbf{1 2 7}$ \\
\hline
\end{tabular}

\begin{tabular}{|c|c|c|c|}
\hline $\begin{array}{c}\text { Tabela 8 - Velocidade da célula de carga por publicação, } \\
\text { conforme o ensaio adotado. }\end{array}$ \\
\hline Velocidade & $\begin{array}{c}\text { Publicações por tipo de ensaio* } \\
\text { Cisalhamento }\end{array}$ & Tração \\
\hline $0,5 \mathrm{~mm} / \mathrm{min}$ & 21 & 5 & 25 \\
\hline $1,0 \mathrm{~mm} / \mathrm{min}$ & 36 & 10 & 43 \\
\hline $2,0 \mathrm{~mm} / \mathrm{min}$ & 4 & 4 & 8 \\
\hline $5,0 \mathrm{~mm} / \mathrm{min}$ & 37 & 1 & 38 \\
\hline mais de 5,0 mm/min & 5 & 1 & 6 \\
\hline não mencionada & & & 7 \\
\hline Total & $\mathbf{1 0 3}$ & $\mathbf{2 1}$ & $\mathbf{1 2 7}$ \\
\hline
\end{tabular}

*Em algumas publicações foram realizados ambos os ensaios, sendo computados no total como apenas uma publicação.

\section{Unidades utilizadas para força de adesão}

Diferentes unidades têm sido utilizadas para quantificar a força de adesão obtida em testes, dentre elas o Megapascal, o Newton ou Quilograma-força e outras. A tabela 7 apresenta a distribuição destas. O Megapascal foi a unidade mais freqüente (111), representando $87 \%$ dos artigos selecionados.

\section{A velocidade de operação da máquina de ensaios}

A tabela 8 apresenta a utilização de diferentes velocidades de operação da máquina de ensaios, conforme o ensaio adotado pelo estudo. Quando o ensaio adotado era de cisalhamento, a velocidade mais freqüente foi de $5,0 \mathrm{~mm} / \mathrm{min}$ (36\%), sendo seguida pela de $1,0 \mathrm{~mm} / \mathrm{min}(35 \%)$ e pela de $0,5 \mathrm{~mm} / \mathrm{min}$ (20\%). Já quando em ensaios de tração, a velocidade mais freqüentemente adotada foi de $1,0 \mathrm{~mm} / \mathrm{min}$ (47\%), seguida pela de $0,5 \mathrm{~mm} / \mathrm{min}(24 \%)$.

\section{Método para avaliação da resina remanescente}

Artun e Bergland ${ }^{1}$ desenvolveram o Índice de Adesivo Remanescente (ARI), que se tornou bastante difundido entre os pesquisadores. A tabela 9 avalia a utilização do ARI, comparando-a com outros ARI modificados e outros métodos. Foi observado que em $73 \%$ dos artigos selecionados foi utilizado algum método para avaliação da resina remanescente. O ARI desenvolvido por Artun \& Bergland $^{1}$ foi utilizado em $40 \%$ dos casos, ao passo que em 38\% se optou pela modificação ou adaptação do ARI original, e em 22\% foi preconizado um outro tipo de avaliação como, por exemplo o local da falha da adesão.

\section{Análise estatística empregada}

A tabela 10 apresenta a análise estatística empregada, sendo distribuída de acordo com a quantidade de grupos comparados. Apenas três artigos não apresentaram análise estatística dos resultados obtidos. Além dos resultados expostos, foi avaliada a utilização da análise de Weibull, que ocorreu em apenas cinco artigos, representando cerca de $4 \%$ da amostra estudada. 
Tabela 9 - Utilização de método para avaliação da resina remanescente.

Método utilizado

Número de Publicações

ARI original

37

ARI modificado

35

Outro método

21

Não realizado

34

Total

\section{DISCUSSÃO}

Fox, McCabe e Buckley ${ }^{6}$ há quase uma década foram incisivos no sentido de demonstrar a necessidade de uma padronização da metodologia dos estudos, por não haver um consenso entre os pesquisadores. Nesta ocasião foram propostas as seguintes sugestões no sentido de se desenvolver um protocolo para futuros trabalhos em Ortodontia:

- Deveriam ser usadas superfícies de pré-molares extraídos em adolescentes por razões ortodônticas

- Os dentes poderiam ser utilizados após um mês e até seis meses depois da extração, devendo ser conservados em água destilada

- Após a colagem, os espécimes deveriam ficar armazenados em água a $37^{\circ} \mathrm{C}$ durante 24 horas

- A descolagem deveria ser realizada em Máquinas Instron ou similares operando a uma velocidade de $0,1 \mathrm{~mm} / \mathrm{min}$.

- Deveria haver cuidado no sentido de garantir que todos os espécimes recebessem a carga de descolagem em direção e sentido similares.

- Pelo menos 20 e preferivelmente 30 espécimes deveriam ser utilizados por teste

- O sítio da falha adesiva deveria ser citado

- A análise estatística deveria incluir uma análise de sobrevivência para fornecer uma predição do desempenho do material, que pode ser relacionada à situação clínica.

\begin{tabular}{|c|c|c|}
\hline \multicolumn{2}{|c|}{ Tabela 10 - Análise estatística empregada nos artigos. } \\
\hline Análise Estatística & Número de grupos testados por artigo \\
\hline ANOVA/Multiple Range Test & 10 & Três ou mais \\
\hline Teste t & 11 & 82 \\
\hline Teste t e ANOVA & 1 & 9 \\
\hline Kruskal-Wallis & 0 & 1 \\
\hline Mann-Whitney U & 0 & 2 \\
\hline Kruskal + Mann Whitney U & 0 & 4 \\
\hline Wilcoxon & 1 & 2 \\
\hline Sem análise & 1 & 1 \\
\hline Total & $\mathbf{2 4}$ & $\mathbf{2 4}$ \\
\hline
\end{tabular}

- A força de adesão poderia ser quantificada tanto em Newtons como em MegaPascais.

Para que seja discutida a validade de uma padronização ou mesmo novas sugestões para a elaboração de um protocolo se fazem necessárias algumas considerações prévias. No que se refere ao tipo de substrato utilizado, os dentes humanos permanentes são utilizados na maioria dos estudos, como observado neste levantamento, apesar da crescente utilização de dentes bovinos em testes de adesão. Os dentes bovinos têm sido considerados uma boa alternativa, já que além de serem mais facilmente obtidos, têm a estrutura do esmalte similar aos dentes humanos. Embora os dentes bovinos possam ser utilizados satisfatoriamente para se estudar a adesão ao esmalte in vitro, os níveis de força de adesão de braquetes ao esmalte bovino são de $21 \%$ a $44 \%$ menores que no esmalte humano, provavelmente devido a cristais mais largos, diferenças na formação e mais defeitos que o esmalte humano ${ }^{11}, 13$. Desta forma, para efeito de padronização, parece mais apropriado que os pré-molares continuem a ser o substrato de eleição para tais estudos.

Com a crescente demanda de tratamento pelos adultos, outros substratos tais como, restaurações em amálgama, cerâmica e ouro, surgem na prática clínica e junto com estes, surgem produtos que possibilitam o atendimento de tais pacientes. 
Diversos estudos avaliaram a força de adesão de braquetes ortodônticos a estas diferentes superfícies. Nestes casos, um ponto importante seria a utilização de braquetes para incisivos centrais por possuírem uma base mais planificada, que se adaptariam mais facilmente às superfícies a serem testadas, que normalmente são planas, já que é incomum a confecção de espécimes com contornos coronários.

A tabela 3 revela a grande freqüência da utilização da água destilada para armazenamento dos espécimes. A utilização de substâncias como o Timol a 0,1\% parece interessante no sentido de preservar os espécimes livres de contaminação, especialmente em períodos mais prolongados, embora existam alguns estudos que demonstraram não haver diferença estatisticamente significante na força de adesão entre intervalos que variem de 3 horas a 6 meses de estocagem ${ }^{10,16}$.

Os ensaios de cisalhamento foram os mais aplicados para análise da força de adesão, em detrimento dos ensaios de tração. Dentre as hipóteses para justificar este achado, talvez esteja a idéia de similaridade que o ensaio de cisalhamento tem com as forças que mais freqüentemente resultam em falhas na adesão de braquetes clinicamente ${ }^{9}$, embora estas sejam de difícil reprodução devido à sua grande complexidade. Outro ponto a ser considerado talvez seja uma maior facilidade na metodologia aplicada, apesar do ensaio de cisalhamento ser muito mais sensível a falhas na confecção dos espécimes que o ensaio de tração ${ }^{8}$.

Outro ponto de grande discussão diz respeito ao número apropriado de espécimes por grupo. No intuito de se generalizar os trabalhos e até de os tornar mais significativos seria interessante que se utilizasse um número mínimo de 20 espécimes por grupo em estudos in vitro, ao passo que trabalhos com amostras menores poderiam ser utilizados para pesquisas de divulgação interna em instituições, não para publicações ${ }^{6,19}$. Neste levantamento foi observada uma grande variação neste ponto, havendo uma distribuição equilibra- da entre os quatro intervalos adotados, sendo que apenas 28\% dos artigos tiveram mais de 20 espécimes por grupo.

A padronização da unidade de medida para a força de adesão é extremamente importante no sentido de facilitar a comparação de resultados. A utilização do Megapascal (MPa), ou de medidas equivalentes como Newtons por milímetro quadrado $\left(\mathrm{N} / \mathrm{mm}^{2}\right)$ ou Mega Newtons por metro quadrado $\left(\mathrm{MN} / \mathrm{m}^{2}\right)$, é mais apropriada por representarem a força por unidade de área necessária para deslocar o braquete de sua posição. Foi observada uma larga utilização do MegaPascal (87\%) como unidade neste presente estudo o que é animador, embora seja importante mencionar que a utilização de Newtows para se quantificar as forças obtidas também é apropriada, desde que as dimensões da base do braquete empregado sejam mencionadas.

Embora não seja um tema muito discutido, a padronização da velocidade de operação da máquina de ensaios é de extrema importância para que possam ser realizadas comparações entre diferentes trabalhos. Neste estudo ficou constatada a enorme variação existente no que se refere à adoção de um valor para esta velocidade. Tal situação é preocupante, pois se constitui numa das principais causas para discrepância entre trabalhos que analisam variáveis similares. Isto ocorre devido a uma tendência que existe de serem obtidos baixos valores de força de adesão ao esmalte quando é adotada uma maior velocidade de operação, ao passo que quando tal velocidade é menor, a tendência é que os valores de força sejam maiores ${ }^{7}$. Fox, Mc Cabe e Buckley ${ }^{6}$ recomendaram que os ensaios em máquinas Instron ou similares fossem conduzidos com uma velocidade de $0,1 \mathrm{~mm} / \mathrm{min}$. Devido a tal valor proposto ser pouco utilizado na literatura, talvez valores variáveis entre 0,1 e $1 \mathrm{~mm} / \mathrm{min}$ representem uma proposta mais razoável para uma padronização universal, o que está de acordo com os resultados obtidos neste estudo, já que se somados os trabalhos que adotaram $0,5 \mathrm{~mm} / \mathrm{min}$ com os que optaram por $1 \mathrm{~mm} / \mathrm{min}$, chegaremos a $55 \%$ (57) dos que realizaram ensaios 
de cisalhamento e $71 \%$ (15) dos de tração.

Uma preocupação de todos os clínicos é manter a superfície do esmalte sem danos após o procedimento de descolagem. Falhas adesivas dentro do adesivo ou na interface adesivo-braquete se tornam mais desejáveis, do que na interface esmalte-adesivo, já que esta última tem sido responsável pelo surgimento de fraturas e fissuras no momento da descolagem ${ }^{4}$. O índice de resina remanescente (ARI) constitui um excelente recurso no sentido de prover informações a respeito da qualidade da adesão do compósito ao dente e do compósito à base do braquete. Neste levantamento foi observada uma grande variação no que se refere à forma como a resina remanescente ou a falha adesiva foi analisada. O método sugerido inicialmente por Artun e Bergland ${ }^{1}$ é interessante por ser simples, prático e largamente difundido em todo o mundo, sendo apropriadamente o mais indicado no sentido de se padronizar os testes de força de adesão in vitro.

Como apresentado na tabela 10 , o Teste $t$ foi o mais utilizado em trabalhos onde foram comparados 2 grupos diferentes (48\%), sendo ainda empregado em 9 artigos para comparar 3 ou mais grupos. $\mathrm{Na}$ realidade, o utilização do Teste t para se comparar mais de 2 grupos representa um equívoco, já que a probabilidade de erro é aumentada, o nível de significância é alterado, e os testes aplicados não são independentes. A Análise de Variância (ANOVA) representa a opção correta em grande parte dos casos onde 3 ou mais grupos são comparados, tendo sido aplicada em 82 artigos $(79,6 \%)$.

Apesar disso, é importante que seja enfatizado que o Teste $t$ e a Análise de Variância não representam a melhor opção em todos os casos. $\mathrm{Na}$ realidade, tais testes são apropriados quando as amostras avaliadas possuem uma distribuição normal, o que não ocorre sempre ${ }^{5}$. Nestas situações a melhor opção é a utilização de testes não-paramétricos, como os de Kruskal-Wallis e de MannWhitney U.

Embora não seja utilizada em larga escala, conforme observado neste estudo, a Análise de Weibull constitui um excelente recurso estatístico. Consiste em elaborar a probabilidade de falha na adesão a partir de diferentes valores de força aplicados no braquete. Seu uso é interessante por fornecer estimativas da performance clínica de diferentes produtos, apresentando informações mais relevantes ao ortodontista, do que simplesmente a menção dos valores médios de força de adesão ${ }^{17}$.

\section{CONCLUSÕES}

Neste estudo, ficou evidenciado que persiste a falta de consenso na metodologia dos testes de força de adesão in vitro em Ortodontia. Apesar de decorridos quase dez anos desde as sugestões realizadas por Fox, McCabe e Buckley ${ }^{6}$, em diversos pontos discutidos fica inerente a necessidade de que seja estabelecida uma padronização. Suas sugestões continuam sendo pertinentes, havendo apenas outras sugestões e algumas modificações já consideradas, relativas à velocidade de operação da máquina de ensaios e ao uso do ARI conforme preconizado por Artun e Bergland ${ }^{1}$, que deveriam ser realizadas no sentido de tornar um possível protocolo mais adaptável aos métodos utilizados por diferentes pesquisadores em todo o mundo.

Um protocolo único continua sendo vital, e seria desejável no sentido de facilitar o preparo dos espécimes, tornar os métodos mais simples e exeqüíveis, ao passo que tornaria os resultados obtidos pelos pesquisadores muito mais compreensiveis e valorosos, facilitando comparações entre materiais em diferentes trabalhos, oferecendo orientações muito mais seguras e práticas ao ortodontista. 


\title{
An analysis of in vitro bond strength testing in Orthodontics
}

\begin{abstract}
The aim of this study was to examine the recent methodology used in laboratory analysis of bond strength in orthodontics. The material used was all the articles published in the American Journal of Orthodontics and Dentofacial Orthopaedics or The Angle Orthodontics in which the bonding between the bracket and several surfaces were evaluated by Instron Universal Testing Machine or similar devices. It was found 127 articles, in which $86 \%$ tested the specimens in shear mode, and 14\% used the tensile technique. Among the studies with human teeth (68\%) premolars were the most frequent (57\%), followed by molars (31\%). According to the number of specimens per group, 28 studies analysed from 6 to 10; 30 from 11 to 15; 33 from 16 to 20; and 36 analyzed more than 20 specimens. According to nature of the storage solution, the most used were distilled water (43\%), followed by $0.1 \%$ Timol (28\%) and $0.9 \%$ saline solution (12\%). With regard to the cross head speed, in 25 studies were of $0,5 \mathrm{~mm} / \mathrm{min}$; in 43 were of $1.0 \mathrm{~mm} / \mathrm{min}$; in 8 was of $2.0 \mathrm{~mm} / \mathrm{min}$; and in 38 was of $5.0 \mathrm{~mm} / \mathrm{min}$. The authors concluded that there was not an agreement in the methodology on those studies, and so there is a potential need of technique standardization.
\end{abstract}

Key words: Composites. Orthodontic Bonding. Orthodontics.

\section{REFERÊNCIAS}

1. ARTUN, J.; BERGLAND, S. Clinical trials with crystal growth conditioning as an alternative to acid-etch enamel pretreatment. Am J Orthod, St. Louis, v. 85, p. 333-340, 1984

2. BISHARA, S. E. et al. Effect of a self-etch primer/adhesive on the shear bond strength of orthodontic brackets. Am J Orthod Dentofacial Orthop, St. Louis, v.119, p. 621-624, 2001.

3. BUONOCORE, M. G. Simple method of increasing the adhesion of acrylic filling materials to enamel surface. J Dent Res, Chicago, v. 34, p. 849-853, 1955.

4. CARTENSEN, W. Clinical results after direct bonding of brackets using shorter etch times. Am J Orthod Dentofacial Orthop, St. Louis, v. 89, no.1, p.70-72, 1986.

5. FOX, N. A.; McCABE, J. F. An easily removable ceramic bracket? Br J Orthod, London, v.19, p.305-309, 1992.

6. FOX, N. A.; MCCABE, J. F; BUCKLEY, J. G. A critique of bond strength testing in orthodontics. Br J Orthod, London, v. 21, p. 33-43, 1994.

7. LINDEMUTH, J. S.; HAGGE, M. S. Effect of universal testing machine crossheadspeed on the shear bond strength and bonding failure mode of composite resin to enamel and dentin. Military Medicine, Erlanger, v. 165, no.10, p. 742-746, 2000.

8. KATONA, T. R. The effects of load location and misalignment on shear/peel testing of direct bonded orthodontic brckets- a finite element model. Am J Orthod Dentofacial Orthop, St. Louis, v. 106, no. 4, p. 395-402, 1994.

9. MILLET, D.T.; McCABE, J. F. Orthodontic bonding with glass ionomer cement : a review. Eur J Orthod, Essex, v.18, no. 4, p. 385-399, 1996.

10. NAGEL, N. J. A materials evaluation of ten direct bonding sys- tems utilizing polycarbonate brackets. Am J Orthod, St. Louis, v. 67, p. $460-461,1975$.

11. NAKAMICHI, I.; IWAKU, M.; FUSAYAMA, T. Bovine teeth as possible substitutes in the adhesion test. J Dent Res, Chicago, v. 62, p.1076-1081, 1983.

12. NEWMAN, G.V. Epoxy adhesives for orthodontic attachments: progress report. Am J Orthod, St. Louis, v. 51, no.12, p. 901912, 1965.

13. OESTERLE, L. J.; SHELLHART, W. C.; BELANGER, G. K. The use of bovine enamel in bonding studies. Am J Orthod Dentofacial Orthop, St. Louis, v.114, no.5, p. 514-519, 1998.

14. RETIEF, D. H.; SADOWSKY, P. L. Clinical experience with the acid etch technique in orthodontics. Am J Orthod, St. Louis, v. 68, no. 6, p. 645-654, 1975.

15. REYNOLDS, I. R. A review of direct orthodontic bonding. $\mathbf{B r} \mathbf{J}$ Orthod, London, v. 2, no. 3, p.171-178, 1975

16. REYNOLDS, I. R.; VON FRAUNHOFER, J. A. Direct bonding of orthodontic brackets, a comparative study of adhesives. $\mathbf{B r} \mathbf{J}$ Orthod, London, v. 3, p.143-146, 1976.

17. SARGISON, J. F.; McCABE, J. F.; MILLETT, D.T. A laboratory investigation to compare enamel preparation by sandblasting or acid etching prior to bracket bonding. Br J Orthod, London, v. 26, p.141-146, 1999.

18. WEBSTER, M. J. et al. The effect of saliva on shear bond strengths of hydrophilic bonding systems. Am J Orthod Dentofacial Orthop, St. Louis, v. 119, p. 54-58, 2001.

19. WELBURY, R. R. et al. Factors affecting the bond strength of composite resin to etched glass ionomer cement. J Dent, Bristol, v.16, p.188-193, 1988.
Endereço para correspondência

Julio Orrico de Aragão Pedra e Cal Neto

Rua Almirante Luis Belart, 190 - Apto. 206

Jardim Guanabara

CEP 21941-100

Rio de Janeiro - RJ

E-mail: jcalneto@bol.com.br 